\title{
Effect of Hydrostatic Pressure on Electronic Structure and Optical Properties of InAs: A First Principle Study
}

\author{
M. NOORAFSHAN* \\ Department of Physics, Faculty of Sciences, University of Hormozgan, Bandar-abbas, Iran \\ (Received December 27, 2019; in final form March 24, 2020)
}

\begin{abstract}
First principle calculations have been performed to investigate the effect of hydrostatic pressure on the electronic structure and optical properties of the narrow band gap InAs compound. The calculations have been done by the full-potential linearized augmented plane wave method, based on the density functional theory utilizing Wien $2 \mathrm{k}$ package. The exchange-correlation potential is treated by the generalized gradient approximation and a combination of modified Becke-Johnson plus local-density approximation functional. Regarding the electronic structure, using the band structure calculations at different pressures, the pressure dependence of band gap energy is calculated. In the case of optical properties, the effect of hydrostatic pressure on the real and imaginary parts of the dielectric function $\varepsilon(\omega)$ is calculated.
\end{abstract}

DOI: 10.12693/APhysPolA.137.1153

PACS/topics: InAs compound, hydrostatic pressure, optical properties, electronic structure

\section{Introduction}

During the last century, III-V semiconductors have attracted considerable attention due to their potential applications in the electronic and optoelectronic devices. Among all III-V semiconductors, InAs $\left(E_{g}=0.42 \mathrm{eV}\right)$ is one of the lowest band gap semiconductors (after InSb $\left.\left(E_{g}=0.24 \mathrm{eV}\right)\right)[1]$. Moreover, due to the small direct band gap, InAs is an important material for a variety of optoelectronic devices including photodetectors and lasers operating in the infrared range $[2,3]$.

The band gap energy and optical properties of materials is tunable by applying the pressure $[4,5]$. The calculation of the pressure dependence of band gap of semiconductors is a long-standing problem in physics. Over the past decades, with the development of strained superlattices, there has been even much interest in the pressure dependence of the band-gap semiconductors. The pressure-dependent optical investigations may be the great relevance, since by tailoring the optical properties of materials, the performance of materials can be improved and their application range can be developed.

The electronic and optical properties of InAs using the first principles calculations are investigated by several groups. Feng et al. investigated the electronic and optical properties of InAs at zero pressure using first principle calculations within generalized gradient approximation (GGA) and calculated the band gap energy and the real and imaginary parts of the dielectric function $\varepsilon(\omega)$ [6]. Namjoo et al. investigated the structural, electronic, and optical properties of InAs, InSb and their ternary alloys $\operatorname{InAs}_{x} \mathrm{Sb}_{1-x}$ within density functional theory using

\footnotetext{
*e-mail: m.nourafshan@hormozgan.ac.ir
}

first principle calculations by employing modified BeckeJohnson plus local-density approximation (mBJ-LDA) and GGA functional and calculated the band gap energy and the real and imaginary parts of the dielectric function $\varepsilon(\omega)$ as a function of concentration $x$. Their calculations showed that minimum band gap energy occurs for As concentration around $x \approx 0.3$. The calculations also showed that the calculated band gap energy and the real and imaginary parts of the dielectric function $\varepsilon(\omega)$ using mBJ-LDA functional are in better agreement with available experimental data than GGA approach [7]. Pressure dependence of band gaps of some binary semiconductors including InAs using an adjusted pseudopotential method investigated by Abid et al. and the linear and quadratic coefficients of pressure dependence of band gap energy were calculated [8]. Even though some of optical properties of InAs have been investigated theoretically and experimentally, but there is no earlier calculations on the effect of hydrostatic pressure on the optical properties of InAs. Besides, pressure dependence of band gap energy of InAs using a more accurate method like mBJ-LDA is also missing. Thus, it would be interesting to investigate the effect of pressure on the band gap energy and optical properties of InAs using mBJ-LDA functional. In this work, we follow two main goals: first - to find the pressure dependence of band gap energy of InAs semiconductor, second - to see how the hydrostatic pressure affects on the real and imaginary parts of the dielectric function $\varepsilon(\omega)$.

This paper is organized as follows: in Sect. 2 the details of calculations method are presented. In Sect. 3, the structural properties and the effect of pressure on the band gap energy and optical properties of InAs are investigated. Finally, the main results of this work are summarized in the last section. 


\section{Computational details}

First principle calculations based on density functional theory have developed the vital part of material investigations [9]. The calculated results in this work are obtained using the highly accurate full potential linearized augmented plane wave (FP-LAPW) method, based on the density functional theory (DFT) as implemented in Wien2k package $[10,11]$. The generalized gradient approximation (GGA) [12] and a combination of modified Becke-Johnson plus local-density approximation (mBJ-LDA) [13] functional are employed to treat the exchange and correlation interactions. It is well known that the original form of GGA and LDA approaches are not adequately flexible for the band gap energy calculations. Whereas, the mBJ-LDA functional, can mimic the orbital potential behavior near the band gap and it is expected to give the accurate states position near the band edge. Besides, as mentioned in the introduction, Namjoo et al. showed that the real and imaginary parts of the dielectric function $\varepsilon(\omega)$ using mBJ-LDA functional are in better agreement with available experimental data than GGA approach [7]. Thus, in this work the mBJ-LDA approach has been developed for the calculations of electronic structure and optical properties that depend mainly on the exactitude of exchange potential but not for the structural properties. For the structural properties, the GGA approach is applied.

The radii of the muffin-tin spheres were chosen as $R_{\text {In }}=1.9$ a.u., $R_{\mathrm{As}}=1.8$ a.u. The parameter of the plane wave cut-off $\left(R_{M T} K_{\max }\right)$, which controls the number of basis functions, is confined to 8.0. The spherical harmonics inside the muffin-tin spheres were expanded up to $l_{\max }=10$. The Fourier expansion of the charge density and the potential is chosen as $12 \sqrt{R y}$. To provide a reliable Brillouin zone integration, a set of $3000 k$ point in the irreducible wedge of the Brillouin zone was used, while denser meshes of $10000 k$-points have been used for the optical properties. All parameters are obtained through the convergence of the total energy in the self-consistent calculations.

All the calculations of this work are performed in the presence of spin-orbit interactions. The spin-orbit coupling of the valence electrons is incorporated in the calculations by means of second variational procedure [14].

\section{Results and discussion}

\subsection{Structural properties}

InAs compound has zinc-blende structure and space group $F-43 m[1]$. The In atom is located at the origin $(0,0,0)$ and the As atom is located at $(1 / 4,1 / 4,1 / 4)$. In order to determine the optimized lattice constant, using the experimental lattice constant as a starting point, the total energies of InAs as a function of volume are calculated. Then, the calculated total energies are fitted to the Murnaghan equation of state [15]. Using the
TABLE I

The calculated lattice constant $a$ within GGA approach.

\begin{tabular}{c|c|c}
\hline \hline \multicolumn{3}{|c}{ Lattice constant $a[\AA]$} \\
\hline This work & Exp. & Other calc. \\
\hline 6.097 & $6.058[1]$ & $6.058[16]$ \\
& & $6.051[17]$ \\
& & $6.192[18]$
\end{tabular}

minimum of energy versus volume curve, the equilibrium lattice constant is obtained. The result is given in Table I together with former theoretical and experimental works.

\subsection{Pressure dependence of the electronic structure and optical properties}

\subsubsection{The electronic band structure}

As mentioned in the introduction, InAs is a narrow direct band gap semiconductor. To investigate the effect of pressure on the electronic properties of InAs, first, the electronic band structure at zero pressure within mBJ-LDA is calculated and shown in Fig. 1a. The calculated electronic band structure shows that InAs is a direct band gap semiconductor with the As $4 p$ and In $5 s$ states as valence and conduction bands and the band gap energy $E_{g}=0.47 \mathrm{eV}$. These results are in good agreement with the available experimental and theoretical data $[1,7]$. To investigate the effect of pressure on the electronic properties of InAs, the electronic band structure at different pressure (including positive and negative) values are calculated within mBJ-LDA approach. The results are shown in Fig. 1b-g. One can notice that InAs is a direct band gap semiconductor, but when applying pressure, its band gap energy changes. The calculated values of the band gap energy of InAs at different pressures are given in Table II. Note that by increasing the pressure the band gap energy increases, whereas by decreasing the pressure the band gap decreases.

The calculated band gap energy as a function of pressure are plotted in Fig. 2. The calculated band gap energies are fitted by the second order polynomials as the following formulae:

$$
E_{g}(P)=0.46+0.11 P+0.01 P^{2} .
$$

Comparison of pressure dependence of band gap energy in the present work with available literature data [8] show that even though the trend of pressure dependence of band gap energy is similar, i.e., by increasing the pressure the band gap energy increases, but the linear and quadratic coefficients of pressure dependence of band gap energy are different. This discrepancy is due to employing the different computational methods. The coefficients in the present work are more accurate since the band gap energy at zero pressure using the obtaining formula in comparison to corresponding value in [8] is in excellent agreement with available experimental data [1]. Hence, our calculations can be viewed as a prediction, which can be tested against experiment in future work. 

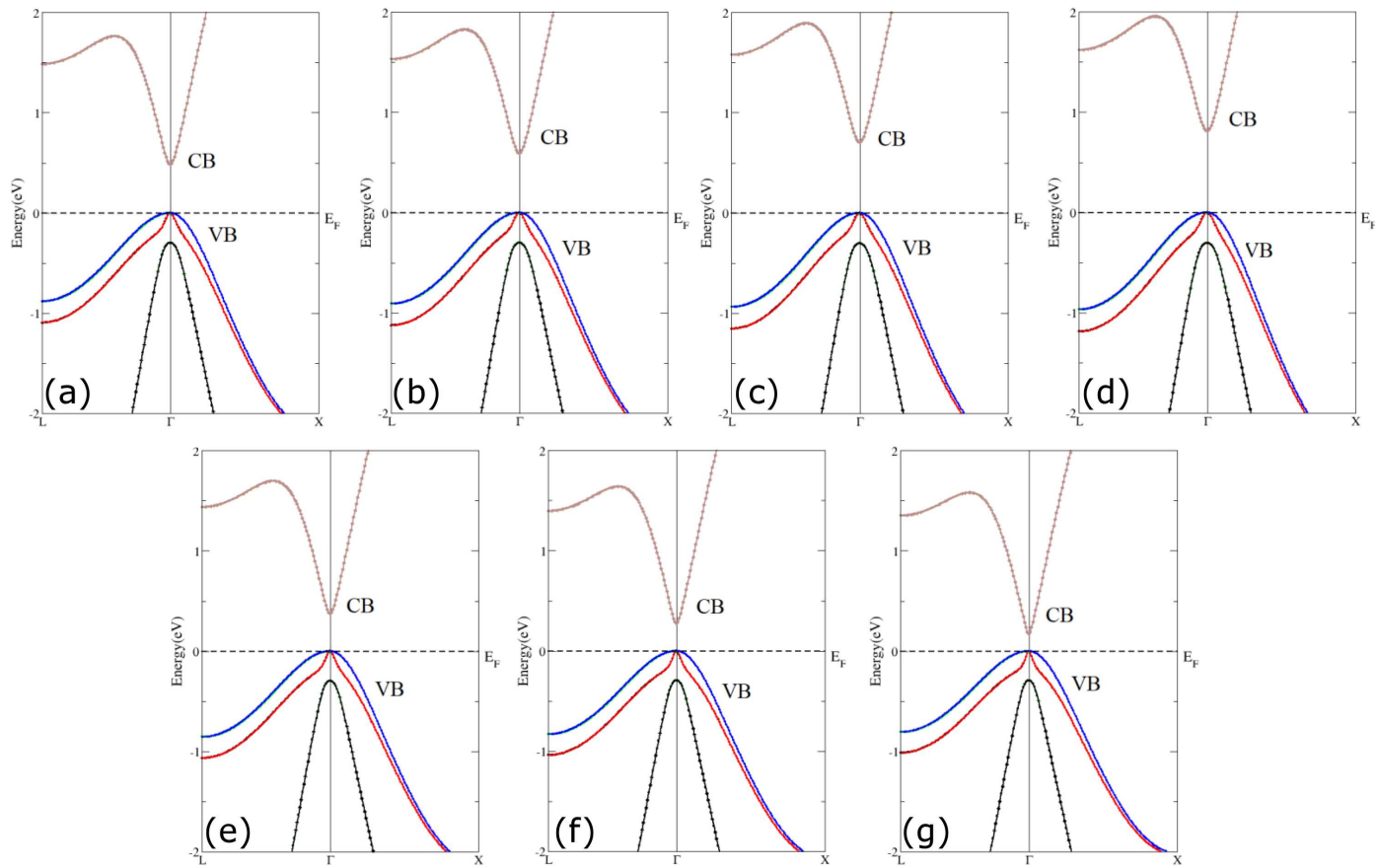

Fig. 1. The band structure of InAs at different pressures using the mBJ-LDA functional: (a) 0 GPa, (b)1.18 GPa, (c) $1.73 \mathrm{GPa}$, (d) $2.29 \mathrm{GPa}$, (e) $-0.88 \mathrm{GPa}$, (f) $-2.67 \mathrm{GPa}$, (g) $-5.32 \mathrm{GPa}$.

TABLE II

The calculated band gap energy $E_{g}$ of InAs at different pressures using the mBJ-LDA functional.

\begin{tabular}{c|c}
\hline \hline $\mathrm{P}[\mathrm{GPa}]$ & $E_{g}[\mathrm{eV}]$ \\
\hline 0 & 0.47 \\
1.18 & 0.58 \\
1.73 & 0.68 \\
2.29 & 0.79 \\
-0.88 & 0.36 \\
-2.67 & 0.25 \\
-5.32 & 0.16
\end{tabular}

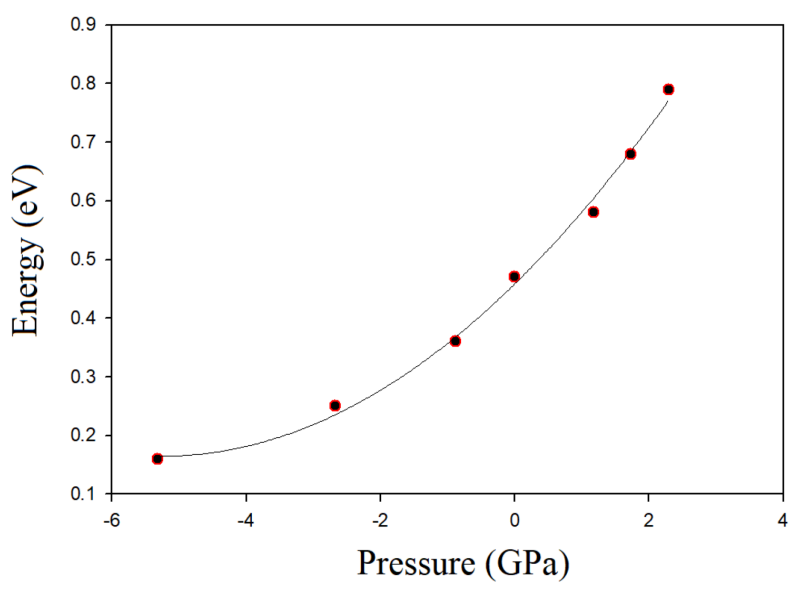

Fig. 2. The calculated band gap energy vs. pressure.

\subsubsection{The optical properties}

The optical properties of materials can be described by means of the transverse complex dielectric function, $\varepsilon(\omega)=\varepsilon_{1}(\omega)+i \varepsilon_{2}(\omega)$, that describes the optical response of the crystal to the electromagnetic field at all energies, and where $\varepsilon_{1}(\omega)$ and $\varepsilon_{2}(\omega)$ are the real and imaginary parts of complex dielectric function respectively. There are two contributions to $\varepsilon(\omega)$ : interband and intraband transitions. The contribution from intraband transitions is only relevant for metals. The interband transition are apportioned to indirect and direct transitions. The indirect interband transitions are related to scattering of photons and are expected to give only a small contribution to $\varepsilon(\omega)$; consequently this contribution is neglected $[19,20]$. The direct interband contributions are considered by calculating the imaginary part of the dielectric function $\varepsilon_{2}(\omega)$. To calculate $\varepsilon_{2}(\omega)$, one must sum over all possible transitions from the occupied valence to the unoccupied conduction band states. By taking the appropriate transition matrix elements into accounts, the imaginary part of the frequency dependent dielectric function within the random phase approximation (RPA) is given by

$$
\begin{gathered}
\varepsilon_{2}(\omega)=\frac{V e^{2}}{2 \pi \hbar^{2} m^{2} \omega^{2}} \int \mathrm{d}^{3} k \sum_{n, n^{\prime}}\left|\left\langle k_{n}|p| k_{n^{\prime}}\right\rangle\right|^{2} \\
\quad \times f\left(k_{n}\right)\left[1-f\left(k_{n^{\prime}}\right)\right] \delta\left(E_{k_{n}}-E_{k_{n^{\prime}}}-\hbar \omega\right),
\end{gathered}
$$

where $\hbar \omega$ is the energy of the incident photon, $p$ is the momentum operator, $\left|k_{n}\right\rangle$ are the eigenfunctions with eigenvalues $E_{k_{n}}$, and $f\left(k_{n}\right)$ is the Fermi distribution function. 


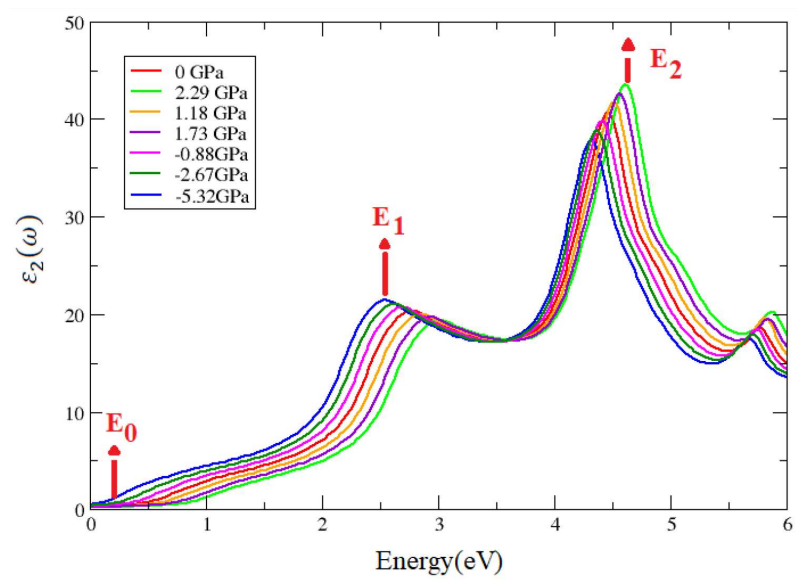

Fig. 3. The calculated imaginary part of dielectric function of InAs at different pressures within mBJ-LDA approach.

The integral is over the first Brillouin zone [20]. The real part of dielectric function is obtained from the imaginary part of dielectric function by use the Kramers-Kronig relation as

$$
\varepsilon_{1}(\omega)=1+\frac{2}{\pi} \int_{0}^{\infty} \mathrm{d} \omega^{\prime} \frac{\varepsilon_{2}\left(\omega^{\prime}\right) \omega^{\prime}}{\left(\omega^{\prime}\right)^{2}-\omega^{2}} .
$$

Since InAs has cubic symmetry, just one dielectric tensor component is needed to calculate to completely characterize the linear optical properties. In order to investigate the effect of hydrostatic pressure on the optical properties of InAs the imaginary part of dielectric function at different pressures are calculated and given in Fig. 3. The calculated imaginary part of dielectric function at zero pressure is in good agreement with available computational data [7]. While the comparison to experimental data shows that even though the general features of the calculated imaginary part of dielectric function is in good agreement with the experimental features but the amplitude of peaks are different from the corresponding experimental ones $[7,21]$. This discrepancy is due to the neglect of local field and excitonic or lifetime broadening.

Here, we focus on the three major points of $\varepsilon_{2}(\omega)$ which are denoted by $E_{0}, E_{1}$ and $E_{2}$. From the electronic band structure calculations, one can identify the transitions that are responsible for the structure in $\varepsilon_{2}(\omega)$. According to the optical matrix elements, it is found that the As $4 p$ and In $5 s$ states play a major role in the optical transitions as the initial and final states. The $E_{0}$ point corresponds to the onset of absorption edge which is corresponding to direct transition from the highest valence band (VB) to the lowest conduction band (CB) at the $\Gamma$ point. The peak $E_{1}$ arises from the transitions between the highest valence bands and the lowest conduction bands around the $L$ point in the Brillouin zone. The peak $E_{2}$ is related to the transition from the highest valence bands to the lowest conduction band near the

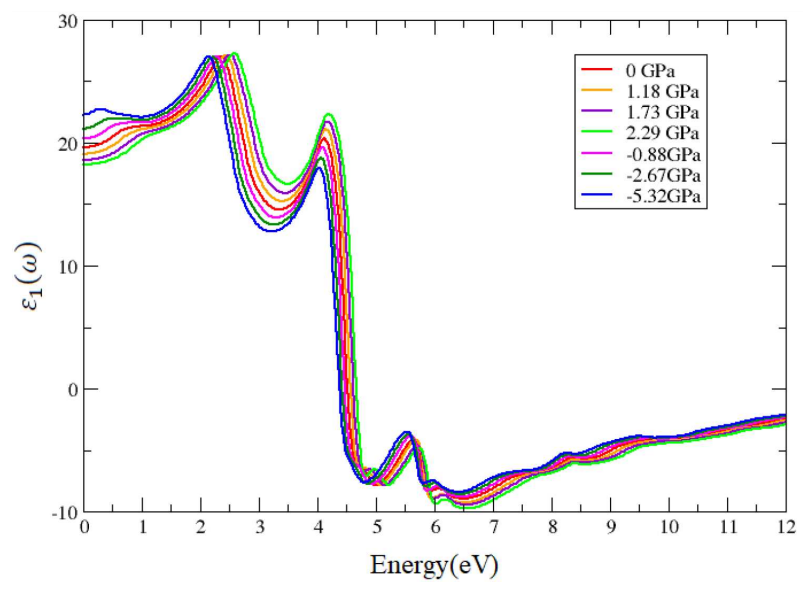

Fig. 4. The calculated real part of dielectric function of InAs at different pressures within mBJ-LDA approach.

$x$ points of the Brillouin zone. As Fig. 3 shows by increasing pressure, the absorption edge is shifted towards higher energies, whereas by decreasing the pressure $E_{0}$ is shifted towards to lower energies.

It can also be seen that by applying pressure the position of major peak $E_{1}$ and $E_{2}$ shifts towards higher energies whereas by decreasing the pressure the position of peak shifts towards lower energies that is in accordance to electronic band structure calculations. As can be seen, although by applying the pressure the positions of peaks shift but these peaks still have the same shape as those of zero pressure.

The real part of dielectric function are calculated at different pressures (Fig. 4). As can be seen from Fig. 4, at all pressures $\varepsilon_{1}(\omega)$ decrease after $4.1 \mathrm{eV}$ and becomes negative in the special energy range, so the incident photon is attenuated and the electromagnetic wave transition through InAs compound is nearly zero in this energy range.

The static dielectric constant $\varepsilon_{1}(0)$ is a very important physical quantity for semiconductors. The values of $\varepsilon_{1}(0)$ at different pressure are given in Table III. These results and those in Fig. 4 indicate that the value of real part of dielectric function near the zero frequency decreases when increasing the pressure, whereas by decreasing the pressure the value of real part of dielectric function increases. Besides, according to the results in Sect. 2, by increasing the pressure the band gap energy increases, whereas by decreasing the pressure the band gap decreases. Thus, this trend is according to the Penn model that shows the inverse relationship between the energy band gap and the real part of static dielectric function as follows [22]:

$$
\varepsilon_{1}(0) \approx\left(\frac{\hbar \omega_{p}}{E_{g}}\right)^{2} .
$$

Therefore, near zero frequency, by decreasing the pressure optical conductivity of InAs increases. In Fig. 5, $\varepsilon_{1}(0)$ as a function of pressure is plotted. The calculated 


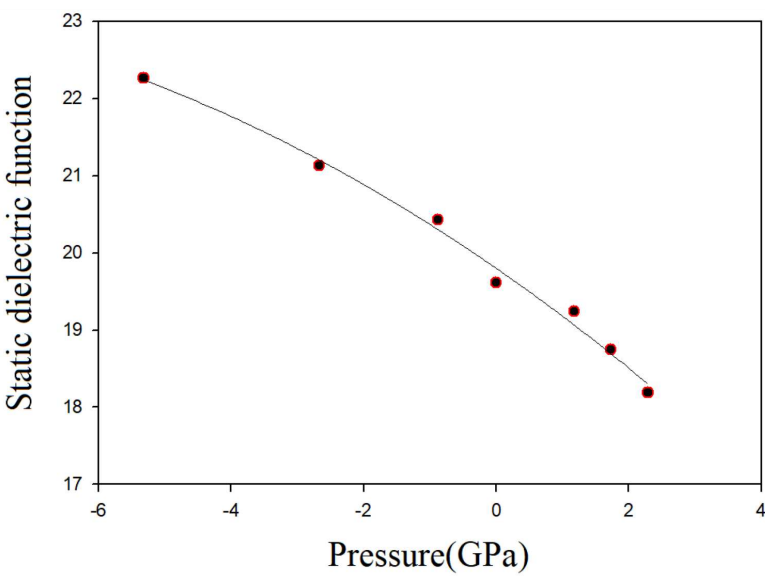

Fig. 5. The calculated static dielectric function versus pressure.

TABLE III

The calculated $\varepsilon_{1}(0)$ at different pressures within mBJ-LDA approach.

\begin{tabular}{c|c}
\hline \hline$P[\mathrm{GPa}]$ & $\varepsilon_{1}(0)$ \\
\hline 0 & 19.61 \\
1.18 & 19.24 \\
1.73 & 18.75 \\
2.29 & 18.19 \\
-0.88 & 20.43 \\
-2.67 & 21.13 \\
-5.32 & 22.26
\end{tabular}

values of $\varepsilon_{1}(0)$ are fitted by the second order polynomials as the following formulae:

$$
\varepsilon_{1}(0)=19.80-0.59 P-0.02 P^{2} \text {. }
$$

There is no available experimental or theoretical data for the pressure dependence of optical properties to compare with. Thus, these results can be viewed as a prediction, which can be tested against experiment in future work.

\section{Conclusion}

In this work, we have studied effect of hydrostatic pressure on the electronic structure and optical properties of InAs compound using the first principles calculations by employing the full potential linearized augmented plane wave (FP-LAPW) method using mBJ-LDA approach. In the case of electronic structure, we find that by increasing the pressure the band gap energy increases, whereas by decreasing the pressure the band gap decreases. Regarding the optical properties, it is found that by increasing pressure the absorption edge and two major peaks $E_{1}$ and $E_{2}$ in the imaginary part of the dielectric function are shifted towards higher energies whereas by decreasing the pressure they are shifted towards to lower energies. The results also show that by decreasing the pressure optical conductivity of InAs increases. These results can be viewed as a prediction, which can be tested against experiment in future work.

\section{References}

[1] O. Madelung, Semiconductors: Data Handbook, Springer, Berlin 2004.

[2] P. Tang, M.J. Pullin, S.J. Chung, C.C. Phyllips, R.A. Stradling, A.G. Norman, Y.B. Li, L. Hart, Semicond. Sci. Technol. 10, 1177 (1995).

[3] W. Dobbelaere, J. De Boeck, P. Heremans, R. Mertens, G. Borghs, W. Luyten, J. Van Landuyt, Appl. Phys. Lett. 60, 3256 (1992).

[4] Z. Nourbakhsh, Physica B 405, 4173 (2010).

[5] H. Salehi, H. Badehian, M. Farbod, Mater. Sci. Semicond. Process. 26, 477 (2014).

[6] Z.B. Feng, H.Q. Hu, S.X. Cui, W.J. Wang, C.Y. Lu, Centr. Europ. J. Phys. 7, 786 (2009).

[7] S. Namjoo, A.S.H. Rozatian, I. Jabbari, P. Puschnig, Phys. Rev. B 91, 205205 (2015).

[8] H. Abid, N. Badi, B. Soudini, N. Amrane, M. Driz, M. Hammadi, H. Aourag, B. Khelifa, Mater. Chem. Phys. 38, 162 (1994).

[9] P. Hohenberg, W. Kohn, Phys. Rev. B 136, 864 (1964).

[10] P. Blaha, K. Schwarz, G. Madsen, D. Kvasnicka, J. Luitz, "WIEN2K: An Augmented Plane Wave plus Local Orbitals Program for Calculating Crystal Properties", Vienna University of Technology, Vienna 2001.

[11] P. Blaha, K. Schwarz, J. Luitz, Comput. Phys. Commun. 59, 399 (1990).

[12] J.P. Perdew, K. Burke, M. Ernzerfhof, Phys. Rev. Lett. 77, 3865 (1996).

[13] F. Tran, P. Blaha, Phys. Rev. Lett. 102, 226401 (2009).

[14] A.H. MacDonald, W.E. Pickett, D.D. Koelling, J. Phys C Solid State Phys. 13, 2675 (1980).

[15] F.D. Murnaghan, Proc. Natl. Acad. Sci. USA 30, 244 (1994).

[16] S. Kalvoda, B. Paulus, P. Fulde, H. Stoll, Phys. Rev. $B$ 55, 4027 (1997).

[17] S.H. Wei, A. Zunger, Phys. Rev. B 60, 5404 (1999).

[18] S.Q. Wang, H.Q. Ye, J. Phys. Condens. Matter 14, 9579 (2009).

[19] N.V. Smith, Phys. Rev. B 3, 1862 (1971).

[20] C. Ambrosch-Draxl, J.O. Sofo, Comput. Phys. Commun. 175, 1 (2006).

[21] D.E. Aspnes, A.A. Studna, Phys. Rev. B 27, 985 (1983).

[22] D.R. Penn, Phys. Rev. B 128, 2093 (1962). 